\title{
Gabor Feature Space Diffusion via the Minimal Weighted Area Method
}

\author{
Chen Sagiv $^{1}$, Nir A. Sochen ${ }^{1}$, and Yehoshua Y. Zeevi ${ }^{2}$ \\ 1 Department of Applied Mathematics \\ University of Tel Aviv \\ Ramat-Aviv, Tel-Aviv 69978, Israel \\ chensagi@post.tau.ac.il, sochen@math.tau.ac.il \\ 2 Department of Electrical engineering \\ Technion - Israel Institute of Technology \\ Technion City, Haifa 32000, Israel \\ zeevi@ee.technion.ac.il
}

\begin{abstract}
Gabor feature space is elaborated for representation, processing and segmentation of textured images. As a first step of preprocessing of images represented in this space, we introduce an algorithm for Gabor feature space denoising. It is a geometric-based algorithm that applies diffusion-like equation derived from a minimal weighted area functional, introduced previously and applied in the context of stereo reconstruction models 6[12. In a previous publication we have already demonstrated how to generalize the intensity-based geodesic active contours model to the Gabor spatial-feature space. This space is represented, via the Beltrami framework, as a $2 D$ Riemannian manifold embedded in a $6 D$ space. In this study we apply the minimal weighted area method to smooth the Gabor space features prior to the application of the geodesic active contour mechanism. We show that this "Weighted Beltrami" approach preserves edges better than the original Beltrami diffusion. Experimental results of this feature space denoising process and of the geodesic active contour mechanism applied to the denoised feature space are presented.

Keywords: Gabor analysis, Geometric-based algorithms, Geodesic active contours, Beltrami framework, Anisotropic diffusion, image manifolds, minimal weighted area method.
\end{abstract}

\section{Introduction}

Textured image segmentation is an important issue in image analysis. However, real world textures are difficult to model. Among the approaches to the analysis of textures are local geometric primitives [9], local statistical features [3], random field models [8,4 and the FRAME theory 23] which combines filtering theory and Markov random field modeling through the maximum entropy principle. Another approach, based on the human visual system has emerged, in which texture features are extracted using Gabor filters [19].

The motivation for the use of Gabor filters in texture analysis is double fold. First, it is believed that simple cells in the visual cortex can be modeled by Gabor 
functions [165, and that the Gabor scheme provides a suitable representation for visual information in the combined frequency-position space [18. Second, the Gabor representation has been shown to be optimal in the sense of minimizing the joint two-dimensional uncertainty in the combined spatial-frequency space [7]. The analysis of Gabor filters was generalized to multi-window Gabor filters [24] and to Gabor-Morlet wavelets 18 24 17/15], and studied both analytically and experimentally on various classes of images [24.

A great deal of attention has been devoted in recent years to the "snakes", or active contour models, which were proposed by Kaas et al [10] for intensity based image segmentation. In this framework an initial contour is deformed towards the boundary of an object to be detected. The evolution equation is derived from minimization of an energy functional, which obtains a minimum for a curve located at the boundary of the object. A major drawback of the classical snakes algorithm is its dependence on the parameterization of the curve. This may actually lead to different results for different choices of parameterization.

The geodesic active contours model [2,11] offers a different perspective for solving the boundary detection problem; It is based on the observation that the energy minimization problem is equivalent to finding a geodesic curve in a Riemannian space whose metric is derived from image contents. The geodesic curve can be found via a parameterization invariant geometric flow. Utilization of the Osher and Sethian level set numerical algorithm [20] allows automatic handling of changes of topology.

It was shown recently that the Gaborian spatial-feature space can be described, via the Beltrami framework 22, as a 4D Riemannian manifold [13] embedded in $\mathbb{R}^{6}$. Based on this approach, the intensity based geodesic active contours method was generalized to the Gabor-feature space of images [21]. It was shown that the geodesic snakes mechanism can be used for texture segmentation when applied to the Gabor spatial feature space of images rather than the intensity images themselves. The metric introduced in the Gabor space was used to derive the inverse edge indicator function $E$, which attracts in turn the evolving curve towards the boundary in the geodesic snakes schemes. Once the Gabor feature space of an image is derived, the scale and orientation for which the maximum amplitude of the transform was obtained are kept for each pixel. Thus, for each pixel, the maximum value of the Gabor transform coefficient and the orientation and scale that yield this maximum value are obtained. This approach results in a $2 D$ manifold embedded in a $6 D$ space. It was shown that using this approach the geodesic snakes yield good results when the textures are homogeneous and can be characterized by these maximum values.

However, the maximum values provide only partial information regarding image structure in the full Gabor feature space. This may, in turn, generate less than satisfactory results in case of more complex textures. One solution to this problem is to apply the geodesic snakes mechanism to the complete Gabor feature space and interpret the Gabor transform of an image as a function assigning for each pixel's coordinates, scale and orientation, a value. Thus, the Gabor transform of an image may be viewed as a $4 D$ manifold embedded in $\mathbb{R}^{6}$. 
An alternative solution is to improve the results obtained from the $2 D$ manifold embedded in $6 D$ space approach which we aim to achieve here.

We apply the weighted area minimization method to improve the results for the orientations which were determined by searching for the maximum value of the Gabor coefficients. We show that it better preserves edges than the Beltrami smoothing operator.

This paper is organized as follows: In section 2 we briefly review the Bayesian formulation in the context of image processing. In section 3 we describe the geodesic active contours method for intensity images. Next, in section 4 we describe the generation of the Gabor feature space. In section 5 we show how to apply the geodesic snakes mechanism in the Gabor feature space. In section 6 we describe the weighted area minimization method, and finally in section 7 , we provide some preliminary results.

\section{Bayesian Formulation}

The Bayesian approach is useful in finding a compromise between the requirements of fidelity of a given image data, and our a priori knowledge or assumptions regarding the nature of "true" images. Accordingly, we consider an image to be made of an ensemble of interacting systems-i.e. pixels, wherein the gray level of each pixel is a realization of a random process. In other words, the gray level of each pixel is drown from a probability distribution that depends on the value of the given noisy image, as well as on a priori information reflecting assumptions about the structure and properties of natural images. For example, and in particular, the smoothness assumption can be interpreted as the "mean free path" of interactions among the above-mentioned pixel generating systems, resulting in some kind of a weighted averaging in a neighborhood of the pixel. The likelihood of an image, given the noisy data set of an image, is obtained by multiplication of the likelihood functions of all the pixels' gray levels. Given a pixel at the coordinates $\left(x_{i}, y_{i}\right)$, according to Bayes rule

$$
P_{x_{i} y_{i}}\left(I\left(x_{i}, y_{i}\right) \mid I_{0}\left(x_{i}, y_{i}\right)\right)=\frac{P_{x_{i} y_{i}}\left(I_{0}\left(x_{i}, y_{i}\right) \mid I\left(x_{i}, y_{i}\right)\right) P_{x_{i} y_{i}}\left(I\left(x_{i}, y_{i}\right)\right)}{P_{x_{i} y_{i}}\left(I_{0}\left(x_{i}, y_{i}\right)\right)}
$$

and

$$
P\left(I \mid I_{0}\right)=\prod_{i, j \in N \times N} P_{x_{i} y_{i}}\left(I\left(x_{i}, y_{i}\right) \mid I_{0}\left(x_{i}, y_{i}\right)\right),
$$

where $N$ is the size of the image, and in the left hand side of both (1) and (2) we have the posteriori probability distribution of either a pixel value (eq. I) or of the entire image (eq. 2) that we wish to compute; Namely the probability of the gray value $I\left(x_{i}, y_{i}\right)$ (or of $I$ ), given the data $I_{0}\left(x_{i}, y_{i}\right)$ (or $\left.I_{0}\right)$. This distribution is calculated in the right hand side of both (11) and (2) as the probabilities of measuring $I_{0}\left(x_{i}, y_{i}\right)$ (or of $I_{0}$ ), given the "true" image, multiplied by the probability of $I\left(x_{i}, y_{i}\right)(I)$ being the true image. In other words, this second term reflects our prior assumption on the distribution of $I(x, y)$. The denominator 
depends only on $I_{0}\left(x_{i}, y_{i}\right)$ and therefore does not affect the optimization process of $I(x, y)$.

One often assumes a Gibbsian distribution, in which case the conditional probability becomes

$$
P_{x y}(A \mid B)=\exp (-\alpha e(A, B)) .
$$

where $e(A, B)$ is an "energy density". Given this type of conditional probability equation (2) becomes

$$
\begin{aligned}
P\left(I \mid I_{0}\right) & =\prod_{i, j \in N \times N} \frac{P_{x_{i} y_{i}}\left(I_{0}\left(x_{i}, y_{i}\right) \mid I\left(x_{i}, y_{i}\right)\right) P_{x_{i} y_{i}}\left(I\left(x_{i}, y_{i}\right)\right)}{P_{x_{i} y_{i}}\left(I_{0}\left(x_{i}, y_{i}\right)\right)} \\
& =\exp \left(-\alpha \int\left(e\left(I, I_{0}\right)-e\left(I_{0}\right)\right) d x d y\right) .
\end{aligned}
$$

Determining which is the image that maximizes the posteriori probability, is equivalent to the selection of the image that minimizes the energy.

Our study generalizes this framework of the statistical approach to images, by considering the probability distribution of texture features and not only (and in the examples given herewith not at all) of the pixels' gray levels. We also choose somewhat non-standard fidelity term and smoothing term. A special form is assumed such that the two terms collapse into one. The technique is borrowed from recent results in stereo reconstruction models [6]12] and our prior assumption is that textures (and/or other image features) are piecewise uniform.

\section{Geodesic Active Contours}

In this section we review the geodesic active contours method for non-textured images [2]. The generalization of the technique for texture segmentation is described in section 4 .

Let $\mathbf{C}(\mathbf{q}):[0,1] \rightarrow \mathbb{R}^{2}$ be a parametrized curve, and let $I:[0, a] \times[0, b] \rightarrow$ $\mathbb{R}^{+}$be the given image. Let $E(r):\left[0, \infty\left[\rightarrow \mathbb{R}^{+}\right.\right.$be an inverse edge detector, so that $E$ approaches zero when $r$ approaches infinity. Visually, $E$ should represent the edges in the image. Minimizing the energy functional proposed in the classical snakes is generalized to finding a geodesic curve in a Riemannian space by minimizing:

$$
L_{R}=\int E(|\nabla I(\mathbf{C}(q))|)\left|\mathbf{C}^{\prime}(q)\right| d q .
$$

We may see this term as a weighted length of a curve, where the Euclidean length element is weighted by $E(|\nabla I(C(q))|)$. The latter contains information regarding the boundaries within the image. The resultant evolution equation is the gradient descent flow:

$$
\frac{\partial \mathbf{C}(t)}{\partial t}=E(|\nabla I|) k \mathbf{N}-(\nabla E \cdot \mathbf{N}) \mathbf{N}
$$

where $k$ denotes curvature. 
If we now define a function $U$, so that $\mathbf{C}=((x, y) \mid U(x, y)=0)$, we may use the Osher-Sethian Level-Sets approach [20] and replace the evolution equation for the curve $\mathbf{C}$, with an evolution equation for the embedding function $U$ :

$$
\frac{\partial U(t)}{\partial t}=|\nabla U| \operatorname{Div}\left(E(|\nabla I|) \frac{\nabla U}{|\nabla U|}\right) .
$$

A popular choice for the stopping function $E(|\nabla I|)$ is given by:

$$
E(|\nabla I|)=\frac{1}{1+|\nabla I|^{2}}
$$

however, other image-specific functions may be used.

\section{Feature Space and Gabor Transform}

The Gabor scheme and Gabor filters have been studied by numerous researchers in the context of image representation, texture segmentation and image retrieval. A Gabor filter centered at the $2 \mathrm{D}$ frequency coordinates $(U, V)$ has the general form of:

$$
h(x, y)=g\left(x^{\prime}, y^{\prime}\right) \exp (2 \pi i(U x+V y))
$$

where

$$
\begin{gathered}
\left(x^{\prime}, y^{\prime}\right)=(x \cos (\phi)+y \sin (\phi),-x \sin (\phi)+y \cos (\phi)), \\
g(x, y)=\frac{1}{2 \pi \sigma^{2}} \exp \left(-\frac{x^{2}}{2 \lambda^{2} \sigma^{2}}-\frac{y^{2}}{2 \sigma^{2}}\right),
\end{gathered}
$$

$\lambda$ is the aspect ratio between $\mathrm{x}$ and $\mathrm{y}$ scales, $\sigma$ is the scale parameter, and the major axis of the Gaussian is oriented at angle $\phi$ relative to the $\mathrm{x}$-axis and to the modulating sinewave gratings.

Accordingly, the Fourier transform of the Gabor function is:

$$
H(u, v)=\exp \left(-2 \pi^{2} \sigma^{2}\left(\left(u^{\prime}-U^{\prime}\right)^{2} \lambda^{2}+\left(v^{\prime}-V^{\prime}\right)^{2}\right)\right)
$$

where, $\left(u^{\prime}, v^{\prime}\right)$ and $\left(U^{\prime}, V^{\prime}\right)$ are rotated frequency coordinates. Thus, $H(u, v)$ is a bandpass Gaussian with its minor axis oriented at angle $\phi$ from the u-axis, and the radial center frequency $F$ is defined by: $F=U^{2}+V^{2}$, with orientation $\theta=\arctan (V / U)$. Since maximal resolution in orientation is desirable, the filters whose sinewave gratings are cooriented with the major axis of the modulating Gaussian are usually considered $(\phi=\theta$ and $\lambda>1)$, and the Gabor filter is reduced to: $h(x, y)=g\left(x^{\prime}, y^{\prime}\right) \exp \left(2 \pi i F x^{\prime}\right)$.

It is possible to generate Gabor-Morlet wavelets from a single mother-Gaborwavelet by transformations such as: translations, rotations and dilations. We can generate, in this way, a set of filters for a known number of scales, S, and orientations K. We obtain the following filters for a discrete subset of transformations: $h_{m n}(x, y)=a^{-m} h\left(\frac{x^{\prime}}{a^{m}}, \frac{y^{\prime}}{a^{m}}\right)$, where $\left(x^{\prime}, y^{\prime}\right)$ are the spatial coordinates rotated by 
$\frac{\pi n}{K}$ and $m=0 \ldots S-1$. Alternatively, one can obtain Gabor wavelets by logarithmically distorting the frequency axis [18] or by incorporating multiwindows [24. In the latter case one obtains a more general scheme wherein subsets of the functions constitute either wavelet sets or Gaborian sets.

The feature space of an image is obtained by the inner product of this set of Gabor filters with the image:

$$
W_{m n}(x, y)=R_{m n}(x, y)+i J_{m n}(x, y)=I(x, y) * h_{m n}(x, y) .
$$

\section{Application of Geodesic Snakes to the Gaborian Feature Space of Images}

The proposed approach enables us to use the geodesic snakes mechanism in the Gabor spatial feature space of images by generalizing the inverse edge indicator function $E$, which attracts in turn the evolving curve towards the boundary in the classical and geodesic snakes schemes. A special feature of our approach is the metric introduced in the Gabor space, and used as the building block for the stopping function $E$ in the geodesic active contours scheme.

Sochen et al 22] view images and image feature space as Riemannian manifolds embedded in a higher dimensional space. For example, a gray scale image is a $2 D$ Riemannian surface (manifold), with $(x, y)$ as local coordinates, embedded in $\mathbb{R}^{3}$ with $(X, Y, Z)$ as local coordinates. The embedding map is $(X=x, Y=y, Z=I(x, y))$, and we write it, by abuse of notations, as $(x, y, I)$. When we consider feature spaces of images, e.g. color space, statistical moments space, and the Gaborian space, we may view the image-feature information as a $N$-dimensional manifold embedded in a $N+M$ dimensional space, where $N$ stands for the number of local parameters needed to index the space of interest and $M$ is the number of feature coordinates. For example, we may view the Gabor transformed image as a 2D manifold with local coordinates $(\mathrm{x}, \mathrm{y})$ embedded in a $6 \mathrm{D}$ feature space. The embedding map is $(x, y, \theta(x, y), \sigma(x, y), R(x, y), J(x, y))$, where $\mathrm{R}$ and $\mathrm{J}$ are the real and imaginary parts of the Gabor transformed image, and $\theta$ and $\sigma$ as the direction and scale for which a maximal response has been achieved. Alternatively, we can represent the Gabor transform space as a $4 \mathrm{D}$ manifold with coordinates $(x, y, \theta, \sigma)$ embedded in the same $6 \mathrm{D}$ feature space. The embedding map, in this case, is $(x, y, \theta, \sigma, R(x, y, \theta, \sigma), J(x, y, \theta, \sigma))$. The main difference between the two approaches is whether $\theta$ and $\sigma$ are considered to be local coordinates or feature coordinates.

A basic concept in the context of Riemannian manifolds is distance. Consider, for example, we take a two-dimensional manifold $\Sigma$ with local coordinates $\left(\sigma_{1}, \sigma_{2}\right)$. Since the local coordinates are curvilinear, the distance is calculated using a positive definite symmetric bilinear form called the metric whose components are denoted by $g_{\mu \nu}\left(\sigma_{1}, \sigma_{2}\right)$ :

$$
d s^{2}=g_{\mu \nu} d \sigma^{\mu} d \sigma^{\nu},
$$

where we used the Einstein summation convention: elements with identical superscripts and subscripts are summed over. 
The metric on the image manifold is derived using a procedure known as pullback. The manifold's metric is then used for various geometrical flows. We shortly review the pullback mechanism. More detailed information can be found in 22$]$.

Let $X: \Sigma \rightarrow M$ be an embedding of $\Sigma$ in $M$, where $M$ is a Riemannian manifold with a metric $h_{i j}$ and $\Sigma$ is another Riemannian manifold. We can use the knowledge of the metric on $M$ and the map $X$ to construct the metric on $\Sigma$. This pullback procedure is as follows:

$$
\left(g_{\mu \nu}\right)_{\Sigma}\left(\sigma^{1}, \sigma^{2}\right)=h_{i j}\left(X\left(\sigma^{1}, \sigma^{2}\right)\right) \frac{\partial X^{i}}{\partial \sigma^{\mu}} \frac{\partial X^{j}}{\partial \sigma^{\nu}},
$$

where we used the Einstein summation convention, $i, j=1, \ldots, \operatorname{dim}(M)$, and $\sigma^{1}, \sigma^{2}$ are the local coordinates on the manifold $\Sigma$.

If we pull back the metric of a 2D image manifold from the Euclidean embedding space $(\mathrm{x}, \mathrm{y}, \mathrm{I})$ we get:

$$
\left(g_{\mu \nu}(x, y)\right)=\left(\begin{array}{cc}
1+I_{x}^{2} & I_{x} I_{y} \\
I_{x} I_{y} & 1+I_{y}^{2}
\end{array}\right)
$$

The determinant of $g_{\mu \nu}$ yields the expression: $1+I_{x}{ }^{2}+I_{y}{ }^{2}$. Thus, we can rewrite the expression for the stopping term $E$ in the geodesic snakes mechanism as follows:

$$
E(|\nabla I|)=\frac{1}{1+|\nabla I|^{2}}=\frac{1}{\operatorname{det}\left(g_{\mu \nu}\right)} .
$$

We may interpret the Gabor transform of an image as a function assigning to each pixel's coordinates, scale and orientation, a value (W). Next, we get the scale and orientation for which we have received the maximum amplitude of the transform for each pixel. Thus, for each pixel, we obtain: $W_{\max }$, the maximum value of the transform, $\theta_{\max }$ and $\sigma_{\max }$ - the orientation and scale that yielded this maximum value. This approach results in a $2 \mathrm{D}$ manifold (with local coordinates $(x, y)$ ) embedded in a $6 \mathrm{D}$ space (with local coordinates $(x, y, R(x, y), J(x, y), \theta(x, y)$, $\sigma(x, y))$. If we use the pullback mechanism described above we get the following metric:

$$
\left(g_{\mu \nu}\right)=\left(\begin{array}{cc}
1+R_{x}^{2}+J_{x}^{2}+\sigma_{x}^{2}+\theta_{x}^{2} & R_{x} R_{y}+J_{x} J_{y}+\sigma_{x} \sigma_{y}+\theta_{x} \theta_{y} \\
R_{x} R_{y}+J_{x} J_{y}+\sigma_{x} \sigma_{y}+\theta_{x} \theta_{y} & 1+R_{y}^{2}+J_{y}^{2}+\sigma_{y}^{2}+\theta_{y}^{2}
\end{array}\right)
$$

We use the fact that the determinant of the metric is a positive definite edge indicator to determine $E$ as the inverse of the determinant of $g_{\mu \nu}$. Here $g_{\mu \nu}$ is a function of the two spatial variables only $x$ and $y$, therefore, we obtain an evolution of a $2 \mathrm{D}$ manifold in a $6 \mathrm{D}$ embedding space.

\section{Smoothing of the Orientation Data by Application of the Weighted Area Minimization Method}

In the previous section we have described how the Gabor feature space can be treated as a $2 D$ manifold embedded in $6 D$ space. We have used a maximum 
criterion to obtain a single orientation and scale for each pixel location. However, this information does not always well represent the textural information and is sensitive to local variations in the texture characteristics. Therefore, the resultant orientation data can be quite noisy. Also, some random noise can deteriorate the resultant data. Our aim is to reduce the amount of noise in the orientation data and obtain a smoother function to be used in the geodesic snakes mechanism.

We obtain the Gabor feature coefficients as a function of $x, y, \theta(x, y)$ and $\sigma(x, y)$. This discussion is devoted to the manipulation of $\theta$, therefore we select a single scale $\sigma$ and generate a set of Gabor filters for that scale, which differ in their orientation. Thus, the generated Gabor feature space is a function of $x, y, \theta(x, y)$. Our aim is to reduce the amount of noise in $\theta$, whether its source is a heterogeneous texture or some random noise. We define an energy functional which minimizes the magnitude of the Gabor coefficients function weighted by an area element determined by $x, y, \theta(x, y)$.

$$
\mathbf{S}(\theta)=\int D(x, y, \theta) \sqrt{g\left(\theta_{x}, \theta_{y}\right)} d x d y
$$

where

$$
D(x, y, \theta)=\frac{1}{\left(R^{2}+J^{2}+c\right)}
$$

is a data fidelity term and $g$ is the determinant of

$$
\left(g_{\mu \nu}\right)=\left(\begin{array}{cc}
1+\theta_{x}^{2} & \theta_{x} \theta_{y} \\
\theta_{x} \theta_{y} & 1+\theta_{y}^{2}
\end{array}\right) .
$$

The combination $\sqrt{g} d x d y$, an area element of the orientation manifold $(x, y$, $\theta(x, y))$, is the term that forces smoothing as the orientation field reduces its overall area when it flows towards the optimal solution. For trivial data term the gradient descent process is the Beltrami flow that ignores any data edges that are not already very pronounced in the initial noisy guess. On the other hand a trivial metric for the orientation manifold results in decoupling of the different orientation values in different locations as the metric is the only place where derivatives of $\theta$ may appear. This decoupling leads to a simple solution: At each pixel the orientation for which maximum response is achieved is chosen. As we have noted above this leads to a noisy solution that may undermine the correctness of the segmentation process.

The constant $c$ in the denominator has two roles: The first role is merely numeric, to avoid division in zero. The second role has a geometrical meaning since this constant determines the convergence properties of our scheme. If this constant is very small, the evolution depends more on the values of the Gabor transform, $\left(R^{2}+J^{2}\right)$, and the smoothing of $\theta$ is less dominant. If the constant is very large compared with the values of the Gabor transform, then they are less dominant in the evolution, and the smoothing of $\theta$ is the same as in the Beltrami scheme.

Considering the Bayesian formulation we notice that we may rewrite the energy density as $e=(D-$ const $) \sqrt{g}+$ const $\sqrt{g}$. We note that the first term is 
a fidelity term that forces $\theta$ to align according to the orientation in the noisy original image while the second term pushes towards a minimal surface solution. Note that the $\sqrt{g}$ in the first term means that the fidelity term is to be thought of as a function on the orientation manifold. Choosing the same constant for both terms leads to the functional $S$ written above. Note that we do indeed generalize the formalism by considering features, i.e. orientations in this specific implementation, rather than intensity. Our assumption is that images are piecewise continuous with respect to all the relevant image features/attributes. In the case of textureless images, i.e. gray level only, this continuity becomes identical to smoothness.

Thus, we process the manifold $\theta(x, y)$, while obtaining the maximum value for the Gabor coefficients, so that the contribution and impact of each component leads to satisfactory result.

Using the Euler-Lagrange method we obtain the following equation:

$$
\frac{\delta S}{\delta \theta}=-\operatorname{div}\left(\frac{\nabla \theta}{\left(R^{2}+J^{2}\right)(x, y, \theta(x, y)) \sqrt{g}}\right)-\frac{\left(R^{2}+J^{2}\right)_{\theta}(x, y, \theta(x, y)) \sqrt{g}}{\left(R^{2}+J^{2}\right)^{2}(x, y, \theta(x, y))}
$$

According to the steepest descent method the evolution equation for $\theta$ is:

$$
\theta_{t}=-\frac{\delta S}{\delta \theta}
$$

\section{$7 \quad$ Results and Discussion}

The Beltrami flow, being a nonlinear diffusion scheme, offers advantages in processing and analysis of images compared with linear diffusion. In the context of the present study it preserves edges more accurately. We use a similar approach to the Beltrami flow, where the Gaborian orientation data, $\theta$, is treated as a $2 D$ manifold embedded in $3 D$ space, $(x, y, \theta)$. Our aim is to smooth the orientation information when accounting for the maximal Gabor coefficients obtained. Following the minimal weighted area diffusion, we can use $\theta$ as the input to the geodesic snakes algorithm. Geodesic snakes is an efficient geometric flow scheme for boundary detection, where the initial conditions include an arbitrary function $U$ which implicitly represents the curve, and a stopping term $E$ which contains the information regarding the boundaries in the image. We generalize the definition of gradients, usually considered in the context of intensity gradients over $(x, y)$ to other possible gradients in scale and orientation. This gradient information is the input function $E$ to the newly generalized geodesic snakes flow.

Next, we present the results of the minimal weighted area method compared to the Beltrami scheme. [For the complete set of full size images and a demo see the web-page: http://www-visl.technion.ac.il/emmcvpr2001].

In this study we have generated the Gabor wavelets for eight orientations, the scale being kept constant. In the geodesic snakes mechanism $U$ was initiated to be a signed distance function [2]. 


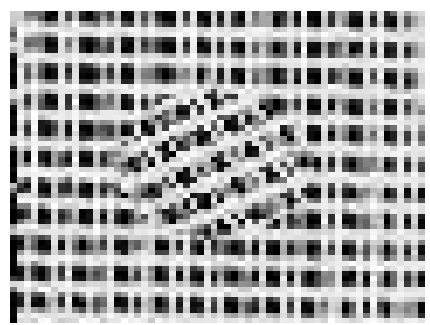

Fig. 1. An image of textures taken from the Brodatz album of textures [1. The circular object is generated from the background texture after rotation by 30 degrees.

The first image (Fig.1) is taken from the Brodatz album of textures [1]. The circular object is generated from the background texture by rotating it by 30 degrees. We apply the Gabor transform to this image and obtain the maximal values of the Gabor coefficients per pixel, and the orientation for which the maximal values were obtained. In figure (2(a)) we see that the orientation information is a piece-wise constant function and that it clearly captures the boundary between object and background. In figure (2(b)) we see the orientation information after random noise was added to it. When the Beltrami flow is applied to the noisy orientation image, if the edges are to be better preserved, we should compromise on the degree of smoothing of the background, as can be seen in figure (2(c)). If further smoothing is desired, the edges are smeared (Fig.2(d)). When the Gabor coefficients are accounted for, we obtain a high degree of smoothing while preserving the sharpness of the edges (Fig.2(e)).

The inter-relations between the Beltrami flow and the weight of the Gabor coefficients can be seen in the next example. By changing the constant value in the denominator from values larger than the mean value of $R^{2}+J^{2}$ (equivalent to the Beltrami flow) to smaller than the mean value we control the impact of the Beltrami numerator to the Gabor denominator.

The second image is similar to the first one, however, here the rotation is done by 45 degrees (Fig. 3). In figure (4)(a)) we see the relevant orientation data after application of the Gabor transform and obtaining the maximal values of the Gabor coefficients per pixel. As we did before, we add random noise to the orientation information [4(b)), and apply the smoothing procedures to remove it. Next, we evaluate the effect of changing the constant value. When the constant is big in comparison to the average value of $R^{2}+J^{2}$, the weighted minimal area method is equivalent to the Beltrami flow (Fig.4(c)). As we decrease this constant the impact of the Gabor coefficients is more evident and we obtain the same degree of smoothing without damaging the edges (Fig.4(d,e)) . This is because the weighing of the Gabor coefficients in the Beltrami flow tends to keep the edges better than when applying the original Beltrami flow, where the only constraint is on the smoothing of the $\theta$ manifold. However, when the constant value is in the range of $R^{2}+J^{2}$, the Gabor coefficients are very dominant comparing to the smoothing of the $\theta$ manifold, and the evolution of $\theta$ can be 

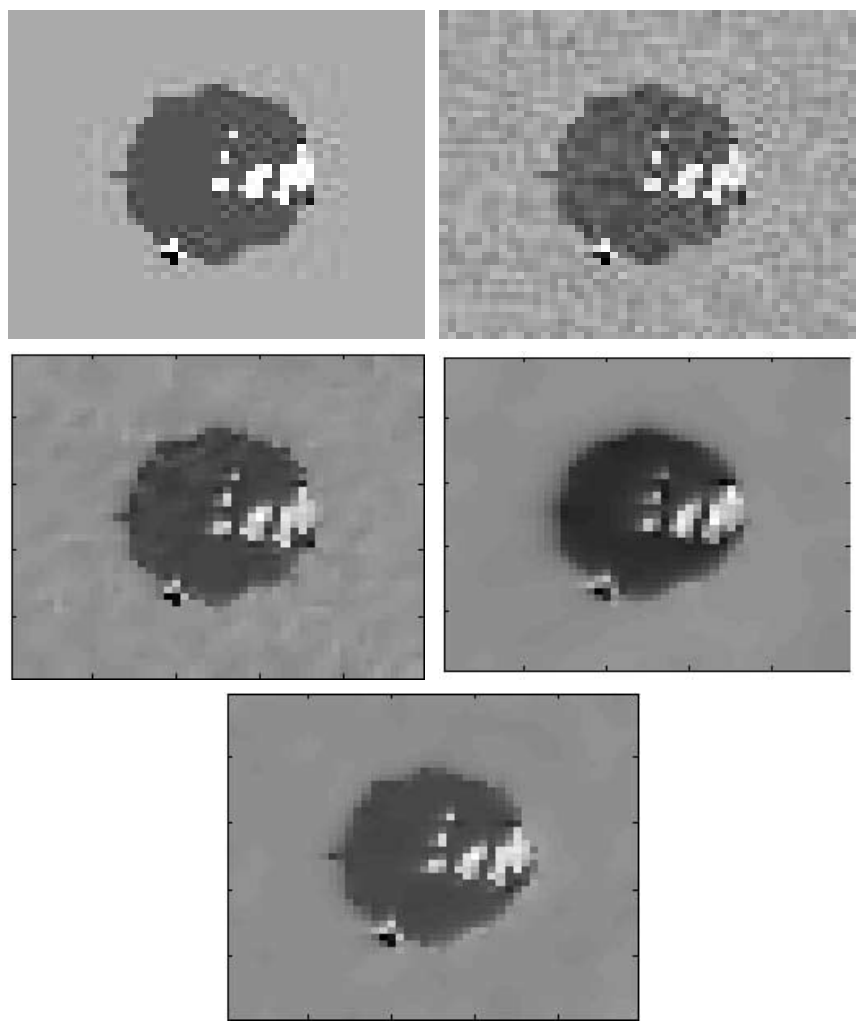

Fig. 2. a. The original orientation information following the application of the Gabor transform and using the maximum criteria (top left). b. The orientation information following addition of random noise (top right). c. Results of the Beltrami diffusion when the process is halted so that significant edges are still evident (middle left). d. If further smoothing is desired, the edges are smeared when the Beltrami diffusion is applied (middle right). e. The result obtained following application of the minimal weighted area method (bottom).

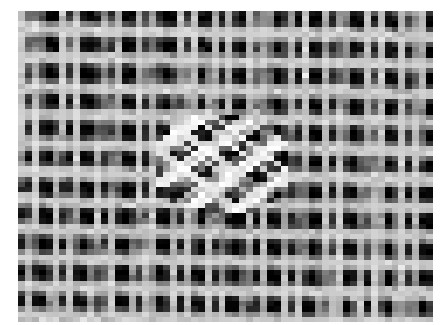

Fig. 3. This image is taken from the Brodatz album of textures [1]. The circular object is generated from the background texture by rotating it by 45 degrees. 

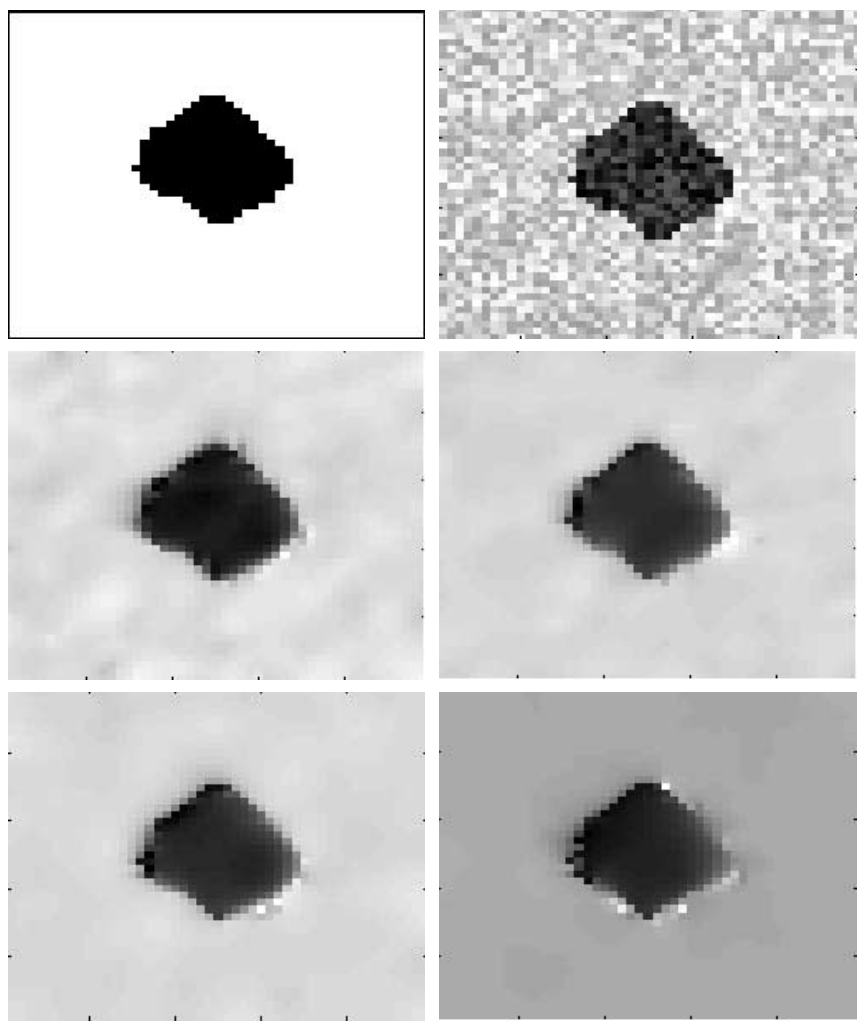

Fig. 4. a. The original orientation information following the application of the Gabor transform and using the maximum criteria (top left). b. The orientation information following addition of random noise (top right). The results of application of the weighted minimal area method are presented, where the mean value of $R^{2}+J^{2}$ is about 200 . The difference between the results is the value of the constant in the denominator. c. $c$ $=10,000$ (middle left). d. $\mathrm{c}=800$ (middle right). e. $\mathrm{c}=600$ (bottom left). f. $\mathrm{c}=200$ (bottom right).

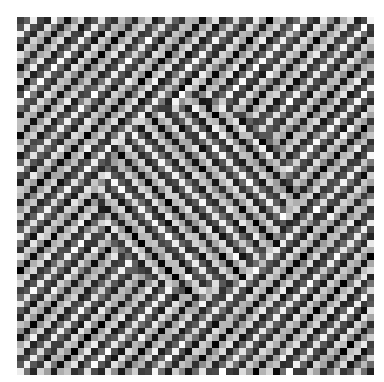

Fig. 5. This image is a synthesized texture composed of linear combination of spatial sinewave gratings of different orientations where some random noise was added to it. 

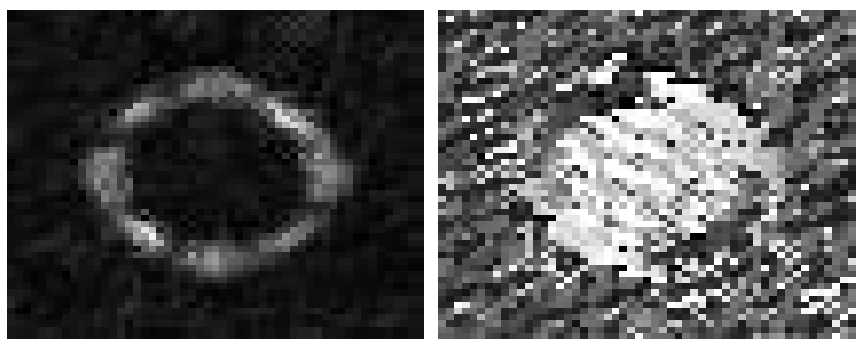

Fig. 6. The maximal values of the Gabor coefficients per pixel are obtained along with the relevant orientation information. a. The magnitude of the Gabor coefficients (left). b. The orientation information (right).

led to local minima. This is manifested in the white dots that appear when the constant is equal to 200 (Fig.4(f)).

In the next example, we demonstrate how the different smoothing processes affect the results of the geodesic snakes mechanism. The original image is a synthesized texture composed of linear combination of spatial sinewave gratings of different orientations where some random noise was added to it (Fig. [5)).

After application of the Gabor filters the maximal value of the Gabor coefficients per pixel is calculated (Fig.6(a)) and the orientation image obtained is noisy (Fig.6(b)). When the Beltrami flow is applied to the noisy orientation image we obtain a smooth result (Fig.[7(a)). However, the edges are more dominant when the Gabor coefficients are accounted for (Fig.7(b)).

Next we use the smoothed $\theta$ obtained to calculate the stopping term $E$ in the geodesic snakes mechanism. The stopping term obtained following the Beltrami diffusion is seen in figure $(7(\mathrm{c}))$, and the one obtained following the minimal weighted area method can be seen in figure $7(\mathrm{~d}))$. The resultant boundaries obtained can be seen in figure $(7(\mathrm{e}+\mathrm{f}))$. The most evident difference between the two results can be seen on the top right hand side of the boundaries. It is clear that using the minimal weighted area method the edges are better captured and detected.

Currently we expand this study to the other Gaborian features, such as the scale parameter $\sigma$ and the sine grating frequency $F$. We explore the behavior of each parameter when considered separately, and also the coupling between these parameters. Another natural continuation of this work is to apply the results of Kimmel and Sochen 14 in order to obtain a more robust orientation diffusion where the orientations manifold is embedded in $R^{2} \otimes S^{1}$. By properly choosing the local coordinate systems for both manifolds the problem arising from the cyclic nature of angles is addressed.

\section{References}

1. P. Brodatz, Textures: A photographic album for Artists and Designers, New York, NY, Dover, 1996. 

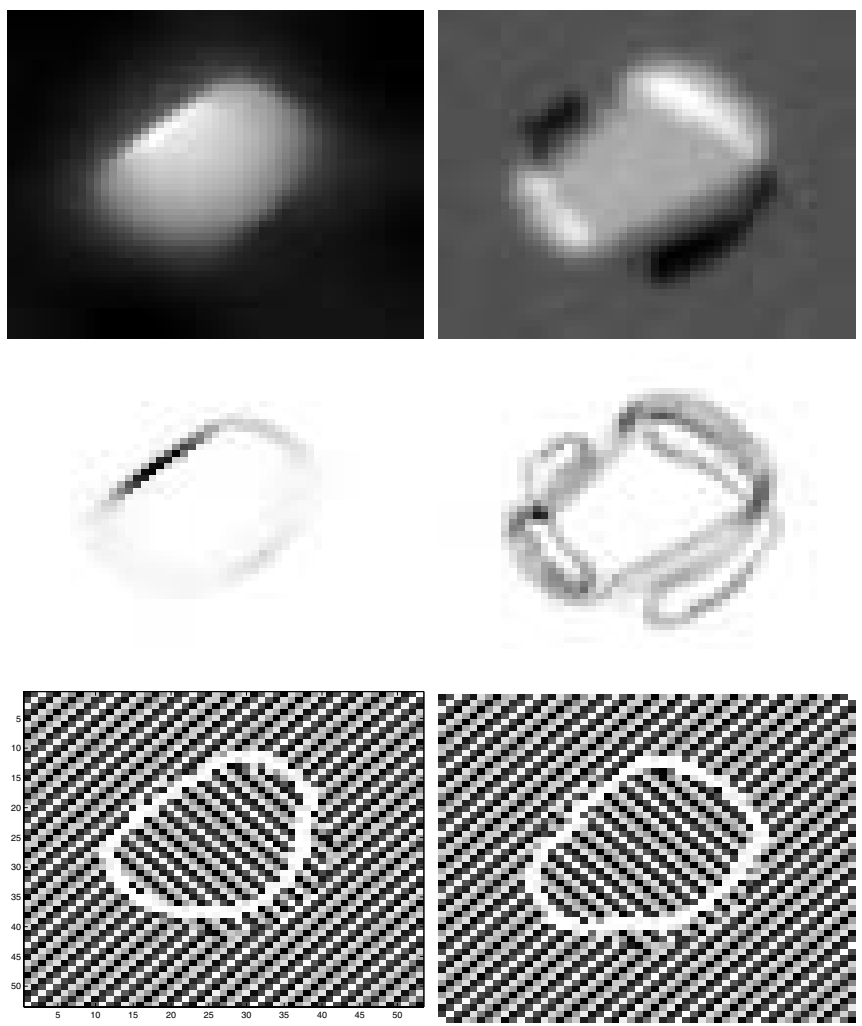

Fig. 7. The orientation data following: a. The Beltrami diffusion (top left), b. The minimal weighted area method (top right). The stopping term obtained when using: c. The Beltrami diffusion (middle left), d. The minimal weighted area method (middle right). The resultant boundary following the application of the geodesic snakes using: e. The Beltrami diffusion(bottom left). f. The minimal weighted area method (bottom right).

2. V. Caselles and R. Kimmel and G. Sapiro, "Geodesic Active Contours", International Journal of Computer Vision, 22(1), 1997, 61-97.

3. R. Conners and C. Harlow "A Theoretical Comparison of Texture Algorithms"IEEE Transactions on PAMI, 2, 1980, 204-222.

4. G.R. Cross and A.K. Jain, "Markov Random Field Texture Models" IEEE Transactions on PAMI, 5, 1983, 25-39.

5. J.G. Daugman, "Uncertainty relation for resolution in space, spatial frequency, and orientation optimized by two-dimensional visual cortical filters", J. Opt. Soc. Amer. 2(7), 1985, 1160-1169.

6. O. Faugeras and R. Keriven, "Variational Principles, surface evolution PDE's, level set methods, and the stereo problem ",IEEE Trans. on Image Processing, 7(3), (1998) 336-344.

7. D. Gabor "Theory of communication" J. IEEE , 93, 1946, 429-459. 
8. S. Geman and D. Geman "Stochastic relaxation, Gibbs distribution and the Bayesian restoration of images", IEEE Transactions on PAMI, 6, 1984, 721-741.

9. B. Julesz "Texton Gradients: The Texton Theory Revisited", Biol Cybern, 54, (1986) 245-251.

10. M. Kaas, A. Witkin and D. Terzopoulos, "Snakes: Active Contour Models", International Journal of Computer Vision, 1, 1988, 321-331.

11. S. Kichenassamy, A. Kumar, P. Olver, A. Tannenbaum and A. Yezzi, "Gradient Flows and Geometric Active Contour Models", Proceedings ICCV'95, Boston, Massachusetts, 1995, 810-815.

12. R. Kimmel, "3D Shape Reconstruction from Autostereograms and Stereo", Special issue on PDEs in Image Processing, Computer Vision, and Computer Graphics, Journal of Visual Communication and Image Representation. In press..

13. R. Kimmel, N. Sochen and R. Malladi, "On the geometry of texture", Proceedings of the 4th International conference on Mathematical Methods for Curves and Surfaces, St. Malo, 1999.

14. R. Kimmel and N. Sochen. "Orientation Diffusion or How to Comb a Porcupine ? ", Special issue on PDEs in Image Processing, Computer Vision, and Computer Graphics, Journal of Visual Communication and Image Representation. In press.

15. T.S. Lee, "Image Representation using 2D Gabor-Wavelets", IEEE Transactions on PAMI, 18(10), 1996, 959-971.

16. S. Marcelja, "Mathematical description of the response of simple cortical cells", $J$. Opt. Soc. Amer., 70, 1980, 1297-1300.

17. J. Morlet, G. Arens, E. Fourgeau and D. Giard, "Wave propagation and sampling theory - part 2: sampling theory and complex waves", Geophysics, 47(2), 1982, 222 -236 .

18. M. Porat and Y.Y. Zeevi, "The generalized Gabor scheme of image representation in biological and machine vision ", IEEE Transactions on PAMI, 10(4), 1988, 452468.

19. M. Porat and Y.Y. Zeevi, "Localized texture processing in vision: Analysis and synthesis in the gaborian space", IEEE Transactions on Biomedical Engineering, 36(1), 1989, 115-129.

20. S.J. Osher and J.A. Sethian, "Fronts propagating with curvature dependent speed: Algorithms based on Hamilton-Jacobi formulations “, J of Computational Physics, 79, 1988, 12-49.

21. C. Sagiv, N. Sochen, and Y.Y. Zeevi , "Gabor Space Geodesic Active Contours", G. Sommer, Y.Y. Zeevi (Eds.), Algebraic Frames for the Perception-Action Cycle, Lecture Notes in Computer Science, Vol. 1888, Springer, Berlin, 2000.

22. N. Sochen, R. Kimmel and R. Malladi , "A general framework for low level vision", IEEE Trans. on Image Processing, 7, (1998) 310-318.

23. S.C. Zhu, Y.N. Wu and D.B. Mumford, "Equivalence of Julesz ensembles and FRAME models", International Journal of Computer Vision, 38(3), 2000, 247265.

24. M. Zibulski and Y.Y. Zeevi, "Analysis of multiwindow Gabor-type schemes by frame methods", Applied and Computational Harmonic Analysis, 4, 1997, 188-221. 